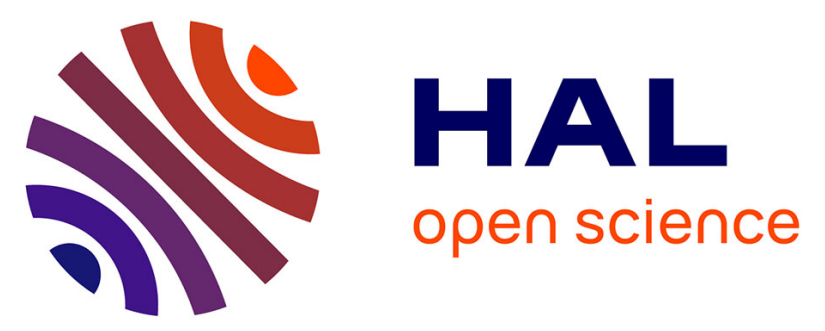

\title{
Importance of chromophore environment on the near infrared absorption of polymeric waveguides
}

Anne-Claire Le Duff, Vincent Ricci, Tomas Pliska, Michael Canva, George I. Stegeman, Kwok Pong Chan, Robert Twieg

\section{- To cite this version:}

Anne-Claire Le Duff, Vincent Ricci, Tomas Pliska, Michael Canva, George I. Stegeman, et al.. Importance of chromophore environment on the near infrared absorption of polymeric waveguides. Applied optics, 2000, 39 (6), pp.947-953. hal-00668291

\section{HAL Id: hal-00668291 \\ https://hal-iogs.archives-ouvertes.fr/hal-00668291}

Submitted on 9 Feb 2012

HAL is a multi-disciplinary open access archive for the deposit and dissemination of scientific research documents, whether they are published or not. The documents may come from teaching and research institutions in France or abroad, or from public or private research centers.
L'archive ouverte pluridisciplinaire HAL, est destinée au dépôt et à la diffusion de documents scientifiques de niveau recherche, publiés ou non, émanant des établissements d'enseignement et de recherche français ou étrangers, des laboratoires publics ou privés. 


\title{
Importance of chromophore environment on the near-infrared absorption of polymeric waveguides
}

\author{
Anne-Claire Le Duff, Vincent Ricci, Tomas Pliska, Michael Canva, George I. Stegeman, \\ K. Pong Chan, and Robert Twieg
}

\begin{abstract}
The near-infrared absorption of two chromophore functionalized polymers and combinations of seventeen different guest chromophores in seven different organic polymer matrices were investigated to assess the effect of chromophore structure and environment on absorption. The near-infrared absorption losses were found to be dramatically larger by as much as 2-3 orders of magnitude in polymer matrices than in solution. Furthermore, the absorption of the long-wavelength tail appears to be related to the glass transition temperature of the polymer matrix that contains the chromophore. These results are interpreted in terms of inhomogeneous broadening. (C) 2000 Optical Society of America
\end{abstract}

OCIS codes: $\quad 160.4330,160.5470,130.3130,300.1030,300.6170,310.3840$.

\section{Introduction}

Polymer waveguides have proved to be successful in quite a number of areas of photonics, most notably as passive polymer fibers. ${ }^{1,2}$ Chromophore-doped systems have also been investigated for many years and are now reaching application levels. ${ }^{3}$ To take best advantage of the high nonlinearity of organic chromophores, the trade-off between the nonlinear optical coefficient and absorption has been extensively studied. However, this has almost invariably been done only in isotropic liquid solution by taking into account the position of the absorption maximum $\lambda_{\max }$ and its absorption cross section. ${ }^{4}$ Only a few investigations have taken any account of the role of the width of the absorption band. Here we demonstrate the influence of the chromophore structure and its environ-

When this research was performed, A.-C. Le Duff, V. Ricci, T. Pliska, M. Canva, and G. I. Stegeman were with the Center for Research and Education in Optics and Lasers, School of Optics, University of Central Florida, 4000 Central Florida Boulevard, Orlando, Florida 32816-2700. A.-C. Le Duff and M. Canva (michael.canva@iota.u-psud.fr) were on leave from the Laboratoire Charles Fabry, Institut d'Optique Théorique et Appliquée, Centre National de la Recherche Scientifique, Université d'Orsay Paris Sud, 91403 Orsay, France. K. P. Chan is with the Molecular OptoElectronics Corporation, 877 25th Street, Watervliet, New York 12189. R. Twieg is with the Department of Chemistry, Kent State University, Kent, Ohio 44242.

Received 28 May 1999; revised manuscript received 29 November 1999 .

0003-6935/00/060947-07\$15.00/0

(C) 2000 Optical Society of America ment on the magnitude of the infrared tail of the absorption band. Some intriguing and important trends are revealed. These results are of primary importance for the optimization of polymeric materials for integrated-optics components that are used either in electro-optic or cascaded all-optical devices at near-infrared wavelengths $(750-900 \mathrm{~nm})$ or in the infrared telecommunication windows (1550 and 1300 $\mathrm{nm}){ }^{5}$ Here, we choose to describe the results as a function of wavelength, in accordance with our applications oriented approach.

Previously published data ${ }^{6}$ showed that the wavelength dispersion of the absorption in the side-chain polymer (SCP) DANS, measured either by thin-film transmission in the visible or waveguide loss in the near infrared, could fit well with a Voigt profile (inhomogeneously broadened absorption model) as shown in Fig. 1. This clearly suggests that one can extrapolate the straight-line behavior observed on semilog plots toward longer wavelengths and far beyond what can be classically measured with thin-film transmission measurements. The slope of this line is defined as

$$
\text { slope }=\frac{d\{\log [\alpha(\lambda)]\}}{d \lambda}
$$

where $\alpha(\lambda)$ is the absorption coefficient of the sample (chromophore in solution or polymer matrix) and the slope is expressed in reciprocal nanometers. 


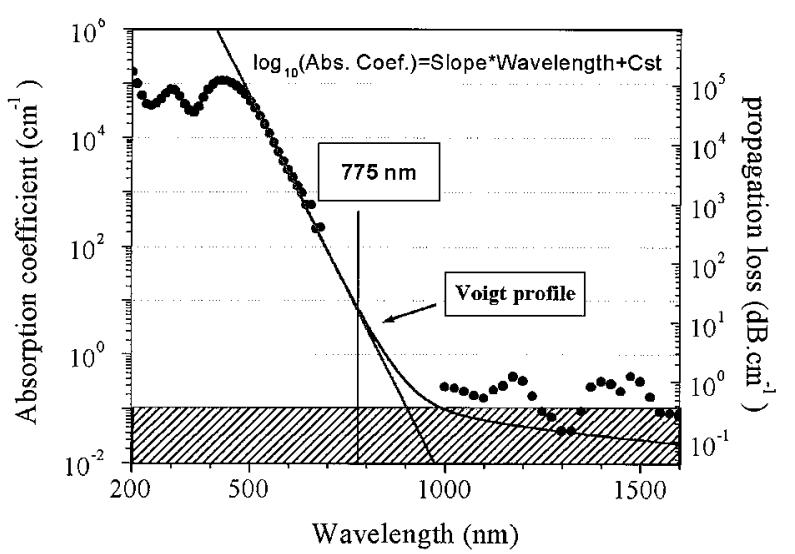

Fig. 1. DANS SCP 1 absorption spectrum derived from thin-film transmission in the visible and waveguide propagation losses in the infrared, fitted to a Voigt profile. ${ }^{6}$ Note that in this representation the linear behavior of the near-infrared tail of the absorption is valid over a spectral range of several hundreds of nanometers and several orders of magnitude in absorption values.

\section{Materials and Characterization}

Our studies have utilized chromophores both covalently bound as side groups to polymers and also as guest molecules dissolved in a polymer matrix. The covalently bound SCP systems studied are DANS SCP 1 and DR1 SCP 2 that were obtained from AkzoNobel and IBM, respectively. These two polymers contain the best known and most widely examined electro-optic chromophores 4-dimethylamino-4'nitrostilbene (DANS 3) and 4-(N-ethyl-N-2hydroxyethyl)-4'-nitroazobenzene (DR1 4). ${ }^{7,8}$ These two chromophores are attached as side groups in polymers 1 and 2 that served as prototype materials for our studies of the near-infrared absorption of various polymers. Their absorption peaks are at 430 and $480 \mathrm{~nm}$, respectively, and Fig. 2 shows their absorption spectra plotted on both a semilog and on a linear scale (inset) as measured in transmission with a spectrophotometer. The main absorption band of DANS SCP 1 is clearly blueshifted relative to DR1 SCP 2. Although the short absorption lengths of the $1-2-\mu \mathrm{m}$-thick polymer films do not permit the measurement of small absorption coefficients, a close inspection of their spectra, in particular on the semilog scale, clearly indicates that DR1 SCP 2 is more transparent in the near infrared than DANS SCP. Note that this behavior could not be observed on the linear scale, where the two absorption curves appear to be superimposed in this region.

We note that data on the infrared absorption tail obtained from DANS SCP samples of different thickness $(0.2-1.7 \mu \mathrm{m})$ also revealed an unexpected sample thickness dependence. Therefore, the protocol employed in this study involves the use of approximately $2-\mu \mathrm{m}$-thick films for all materials and measurements (transmission and propagation). All the data reported in this study were obtained for chemically stable materials whose absorption properties did not evolve with time (on a several month time

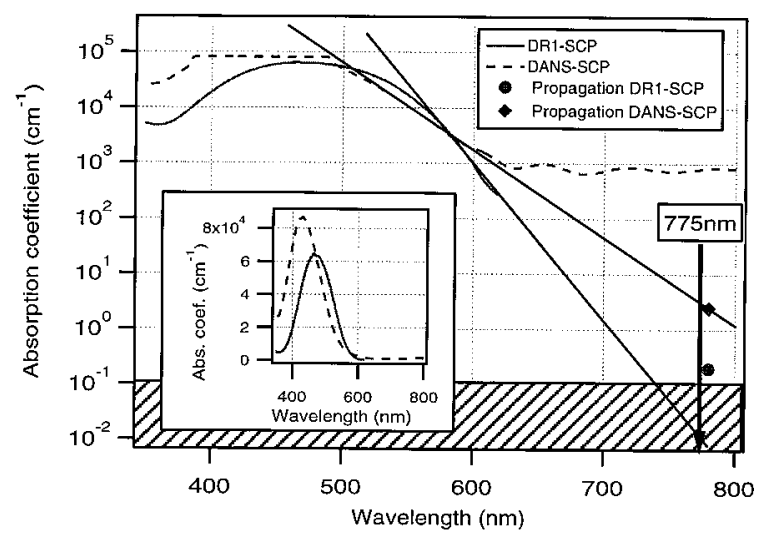

Fig. 2. Absorption spectra of $2-\mu$ m-thick DANS SCP 1 and DR1 SCP 2 films, plotted on both a linear (inset) and a log scale. The weight concentration of active DANS and DR1 chromophore content is $43 \%$ and $24 \%$ of all the polymers, respectively. The straight lines are extrapolations of the absorption curves in the near-infrared region. Propagation loss measurements taken with $2-\mu \mathrm{m}$-thick planar waveguides at $780 \mathrm{~nm}$ are also plotted. A 0.5 - $\mu \mathrm{m}$-thick DANS SCP 1 sample was used to avoid saturation of the absorption coefficient in the linear representation.

scale, stored in the dark at room temperature and in ambient atmosphere). The sample thickness dependent absorption is currently under independent study and will be reported in more detail at a future time.

As is shown in Fig. 2, the extrapolations of the DANS SCP 1 and DR1 SCP 2 absorption to $775 \mathrm{~nm}$ (chosen because this is the harmonic wavelength in cascaded processes at $1.55 \mu \mathrm{m}$ ) yield approximately 2.5 and $0.01 \mathrm{~cm}^{-1}$, respectively $\left(1 \mathrm{~cm}^{-1}=4.34 \mathrm{~dB}\right.$ $\mathrm{cm}^{-1}$ ), a difference of more than 2 orders of magnitude. However, extrapolations that predict absorption values smaller than $0.1 \mathrm{~cm}^{-1}$ are meaningless in waveguides because the propagation losses are typically limited by other factors such as waveguide quality. However, these extrapolations are still useful for comparing materials and structures. In agreement with our extrapolated values, propagation losses in DANS SCP waveguides of approximately 2.5 $\mathrm{cm}^{-1}$ have been experimentally determined. In contrast, for DR1 SCP waveguides attenuation was found to be of the order of $0.2 \mathrm{~cm}^{-1}\left(1 \mathrm{~dB} \mathrm{~cm} \mathrm{~cm}^{-1}\right)$, limited mainly by interfacial scattering losses rather than by absorption. As is discussed below, we further investigated the validity of this extrapolation for some other chromophores.

Because of this strong indication that azobenzene chromophores as found in DR1 SCP appear to have a more attractive loss roll-off in the near infrared, we initiated a systematic study of the importance of the specific chromophore structure and its environment on the near-infrared tail absorption. The structures of the chromophores employed in this study are shown in Fig. 3. Fourteen azobenzene chromophores (5-18) with different donor and acceptor groups were employed in this study. ${ }^{9}$ The azobenzene chromophores have a range of acceptor structure features: 5-11 have a nitrogroup as a primary 


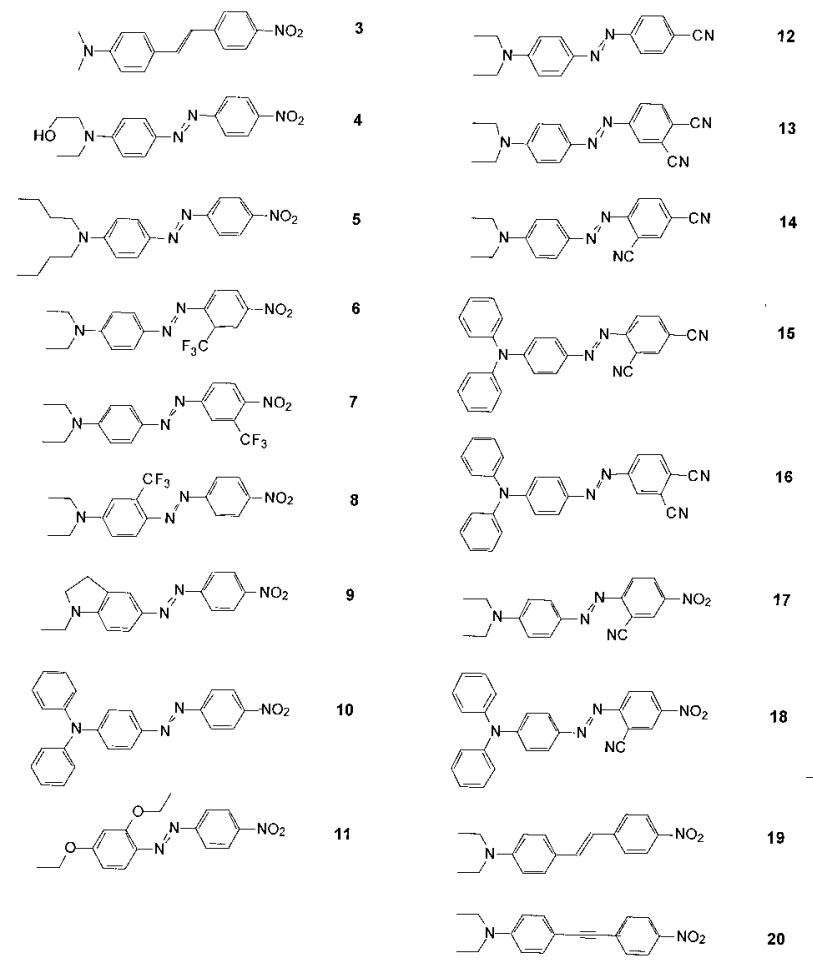

Fig. 3. Chemical structures of the guest chromophores used in this study.

acceptor, 12-16 have a cyanogroup as a primary acceptor, and 17 and 18 have both nitroacceptor and cyanoacceptor groups. These azobenzenes also have a range of donor structure features: all the azobenzenes have aliphatic or cycloaliphatic amine donor substituents except for 10,15,16, and 18, which have arylamine donors, and 11, which has alkoxy donors. One can readily see a number of relationships among the structures: 5, 9, 10, and 11 differ only in the donor with the nitrogroup as the sole common acceptor; 6,7 , and 8 differ only by the location of the trifluoromethyl substituent, whereas the pairs 5 and 10, 13 and 16, 14 and 15 and, finally, 17 and 18, differ only by aliphatic versus aromatic amine donor substituents. The diethylamino analog 19 of the stilbene DANS as well as a tolan (diphenylacetylene) analog 20 are also included in the study.

The absorption spectra of all the dyes were measured both in solution with ethyl acetate as the solvent and also in a guest-host system with poly(methyl methacrylate) (PMMA) A as the matrix. The structures of the host polymers used in this study are shown in Fig. 4. The doped polymer solutions (for the thin films) were prepared with $s$-tetrachloroethane as the solvent. The typical concentration of the dye in the polymer matrix was approximately $4 \%$ by weight. As is shown in Fig. 5 and listed in Table 1, a dramatic difference was observed in the near-infrared tail of the absorption, although the absorption peak in the visible was only slightly shifted and broadened. The slope with which the absorption decreases toward longer wavelengths on a

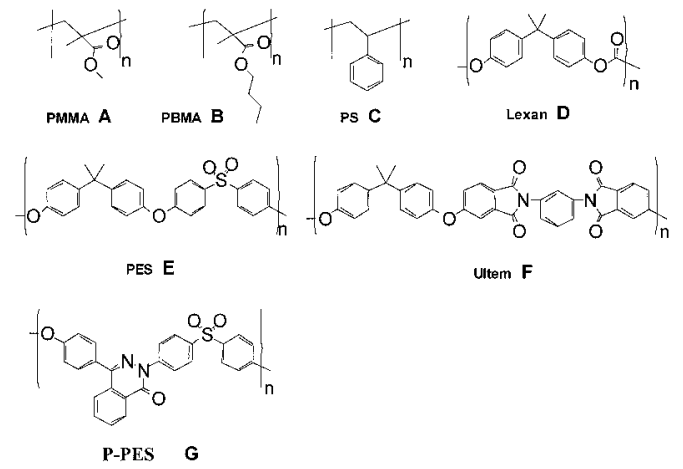

Fig. 4. Chemical structures of the host polymers used in this study.

semilog scale was notably smaller in the solid matrix than in the solution. Altogether, the extrapolated absorption losses at $775 \mathrm{~nm}$ were $2-4$ orders of magnitude larger in the solid compared with the liquid. Specifically, their values are shifted from approximately $10^{-4}-10^{-1} \mathrm{~cm}^{-1}$ in the solvent to approximately $10^{-1}-10^{2} \mathrm{~cm}^{-1}$ in the polymer matrix, normalized to the same weight concentration of chro-
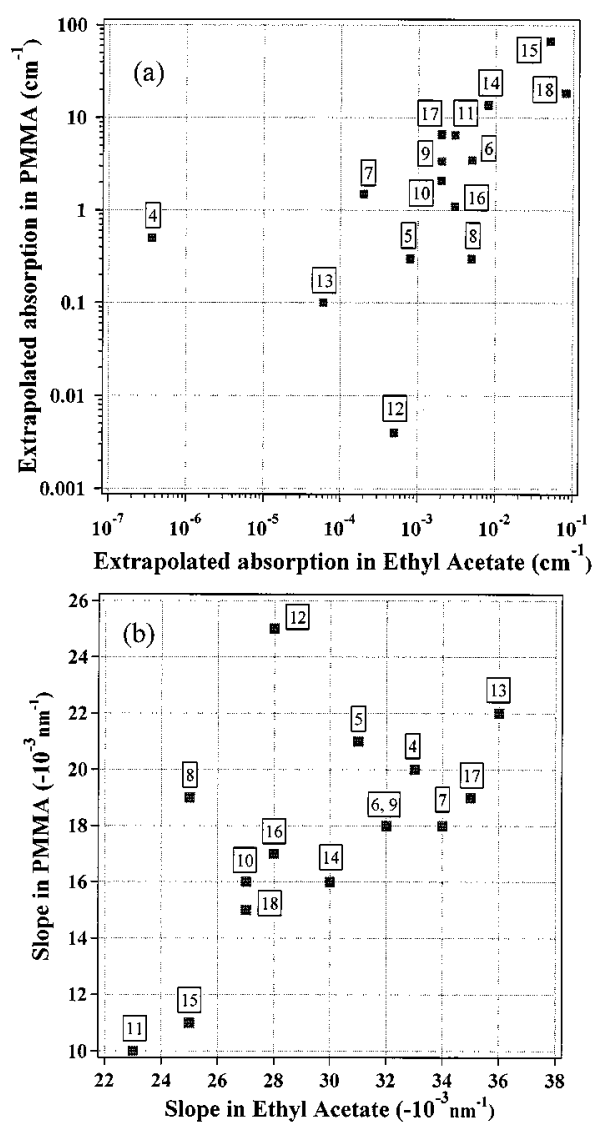

Fig. 5. Effect of environment on the near-infrared absorption tail for a variety of chromophores. (a) Comparison of the absorption coefficient extrapolated to $775 \mathrm{~nm}$ in solution (solvent, ethyl acetate) and a polymer matrix (PMMA). All the data are normalized to a 10 -wt. \% concentration. (b) Correlation between the slope in the absorption tail in ethyl acetate and in PMMA. 


\begin{tabular}{|c|c|c|c|}
\hline \multirow[b]{2}{*}{$\begin{array}{c}\text { Reference } \\
\text { Chromophore }\end{array}$} & \multicolumn{3}{|c|}{ Data Obtained in Ethyl Acetate and PMMA } \\
\hline & $\lambda_{\max }(\mathrm{nm})$ & $\begin{array}{l}\text { Absorption Slope } \\
\left(-10^{-3} \mathrm{~nm}^{-1}\right)\end{array}$ & $\begin{array}{c}\text { Absorption Extrapolated } \\
\text { at } 775 \mathrm{~nm}\left(\mathrm{~cm}^{-1}\right) \\
\text { (Planar Loss whenever Measured) }\end{array}$ \\
\hline 1 DANS SCP (43 wt. $\%$ ) & 430 & 16 & $2.7(2.6)$ \\
\hline 2 DR1-SCP (24 wt. \%) & 469 & 21 & $0.01(0.2)$ \\
\hline 3 DANS & $427 / 427$ & 34/N.A. & $3 \times 10^{-6} / 0.5$ \\
\hline 4 DR1 & $480 / 488$ & $33 / 20$ & $4 \times 10^{-7} / 0.5$ \\
\hline 5 & $486 / 496$ & $31 / 21$ & $8 \times 10^{-4} / 0.3(<0.5)$ \\
\hline 6 & $510 / 516$ & $32 / 18$ & $5 \times 10^{-3} / 3.5$ \\
\hline 7 & $486 / 496$ & $34 / 18$ & $2 \times 10^{-4} / 1.5$ \\
\hline 8 & $463 / 474$ & $25 / 19$ & $5 \times 10^{-3} / 0.3$ \\
\hline 9 & $495 / 506$ & $32 / 18$ & $2 \times 10^{-3} / 3.4$ \\
\hline 10 & $472 / 482$ & $27 / 16$ & $2 \times 10^{-3} / 2.1$ \\
\hline 11 & $400 / 402$ & $23 / 10$ & $3 \times 10^{-3} / 6.5$ \\
\hline 12 & $450 / 458$ & $28 / 25$ & $5 \times 10^{-4} / 4 \times 10^{-3}$ \\
\hline 13 & $490 / 496$ & $36 / 22$ & $6 \times 10^{-5} / 0.1(<0.5)$ \\
\hline 14 & $508 / 518$ & $30 / 16$ & $8 \times 10^{-3} / 13.8$ \\
\hline 15 & $497 / 506$ & $25 / 11$ & $5 \times 10^{-2} / 67$ \\
\hline 16 & $482 / 482$ & $28 / 17$ & $3 \times 10^{-3} / 1.1$ \\
\hline 17 & $531 / 536$ & $35 / 19$ & $2 \times 10^{-3} / 6.6$ \\
\hline 18 & $517 / 528$ & $27 / 15$ & $8 \times 10^{-2} / 18.5$ \\
\hline 19 & $\mathrm{NA} / 448$ & $\mathrm{NA} / 14$ & NA/1.1 (1.2) \\
\hline 20 & NA/424 & NA/17 & $\mathrm{NA} / 0.05(0.7)$ \\
\hline $\begin{array}{l}\text { Average effect on azo } \\
\text { dyes }\end{array}$ & $+8( \pm 3) \mathrm{nm}$ & 1.7 (log scale) & $\times 10^{3}$ \\
\hline
\end{tabular}

${ }^{a}$ The extrapolations are correlated with waveguide propagation losses whenever measured. The results for two SCP systems are provided. All the other data refer to guest-host systems. The values are for a 10-wt. \% chromophore loading. The average effect of the environment is also reported (shift in the position of $\lambda_{\max }$, ratio of the slopes as measured in the solvent and in PMMA, along with the average difference in the extrapolated values of the absorption at $775 \mathrm{~nm}$ in both cases). The last three rows list the results for stilbene and tolan guest dyes. NA, not available.

mophores [Fig. 5(a)]. Such values in the solid are no longer negligible compared to typical waveguide propagation losses that are due to fabrication. Furthermore, one can observe a strong correlation between the slopes when the absorption spectrum is measured in solvent and in PMMA [Fig. 5(b)]. This correlation suggests that a first evaluation of the performance of a new chromophore in a solvent is still a useful diagnostic for comparing the relative merits of different chromophores.

The predictions of the straight-line extrapolation of the absorption toward the near infrared was checked by planar waveguide propagation loss measurements in the SCP's, DANS SCP 1 and DR1 SCP 2, as well as some guest-host systems including the azobenzenes 5 and 13, stilbene 19, and tolan 20 (all in PMMA A). Between 2- and 3- $\mu \mathrm{m}$-thick films were spin coated onto oxidized silicon wafers and cleaved to facilitate end-fire excitation of guided waves. The measurements were performed with a 780-nm diode laser. The light scattered by waveguide imperfections normal to the waveguide was imaged on a CCD camera. The scattered streak exhibits an exponential decay with propagation distance that is due to both scattering and absorptive losses in the waveguide. Such measurements show that the slope extrapolation is valid as long as the decay coefficient exceeds approximately $0.2 \mathrm{~cm}^{-1}\left(1 \mathrm{~dB} \mathrm{~cm}{ }^{-1}\right)$, values below which the scattering propagation losses become dominant. Some of the materials were analyzed in this way, yielding propagation losses ranging from approximately $1 \mathrm{~dB} / \mathrm{cm}$ to several decibels per centimeter, in reasonable agreement with extrapolations of the solid-state absorption data, but well above the values obtained from the solution absorption data. The decay coefficients that we obtained along with their related absorption spectra are sketched in Fig. 2 for the SCP systems, DANS SCP 1 and DR1 SCP 2, and in Fig. 6 for some of the guest-host systems. Excellent agreement was obtained for chromophores 5 and 19. The extrapolated value of the absorption for dye 20 at $775 \mathrm{~nm}$ does not match the loss propagation measurement. It is a typical case for which the loss measurement is dominated by waveguide quality and not by material absorption. The same limitation occurs for the DR1 SCP 2 and to some extent for chromophore 13. But in all cases, the absorption coefficients of the chromophores in PMMA (a glassy polymeric ester) are much larger than the absorption coefficients of the same chromophores in ethyl acetate (a liquid ester).

Such a dramatic impact of the chromophore environment on the near-infrared absorption behavior stimulated a subsequent study of the influence of different organic polymer matrices. Therefore, some of the previous chromophores (namely, 4, 5, and 


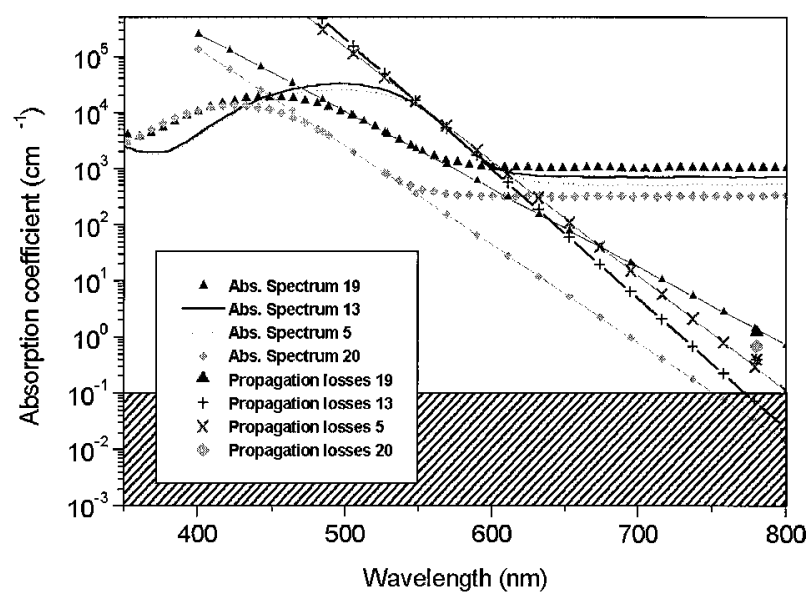

Fig. 6. Absorption spectra in ethyl acetate and propagation loss in a PMMA matrix of the chromophores 5, 13, 19, and 20. All the data are normalized to a 10 -wt. \% concentration. The extrapolated values and the propagation measurements at $775 \mathrm{~nm}$ are in good agreement with the chromophores 5 and 19.

13) were incorporated, still as guest-host systems, in as many as seven different polymer matrices [namely, PMMA A, poly(butyl methacrylate) (PBMA) B, polystyrene (PS) C, BPA-polycarbonate Lexan D, BPA-Polyethersulfone (PES) E, Polyetherimide Ultem $\mathrm{F}$, and the phthalazinone-polyethersulfone ( $\mathrm{P}$ PES) G). ${ }^{10}$ Figure 7 shows the absorption spectra of chromophore 5 in these matrices. The absorption coefficients are normalized to a 10 -wt. \% concentration of the chromophore in the polymer matrix. We observed a strong influence on the chromophore absorption that is due to the polymer environment. The values of the slope differ from one matrix to another, ranging from $-14 \times 10^{-3} \mathrm{~nm}^{-1}$ in PES E to $-21 \times 10^{-3} \mathrm{~nm}^{-1}$ in PMMA A. These different values of the slope result in large differences in the

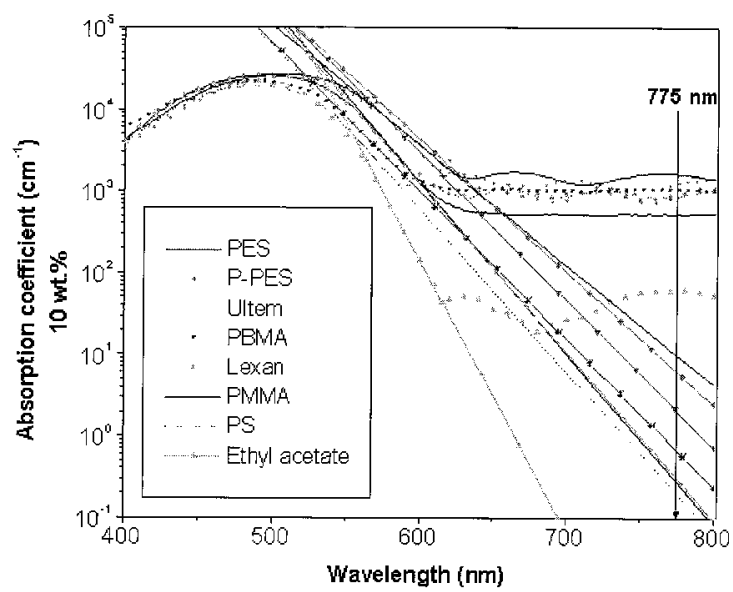

Fig. 7. Absorption spectra of chromophore 5 in different organic polymer matrices and in ethyl acetate solution along with the straight-line extrapolation toward the infrared. Note the different slopes of these extrapolations and the different values they suggest for the absorption in the near infrared, for example, at 775 $\mathrm{nm}$.
Table 2. Glass Transition Temperatures of the Various Polymer Host Matrices

\begin{tabular}{lc}
\hline \multicolumn{1}{c}{ Polymer } & $T_{g}\left({ }^{\circ} \mathrm{C}\right)$ \\
\hline Phthalazinone PES & 303 \\
Ultem (PEI) & 210 \\
PES & 185 \\
Lexan (PC) & 150 \\
DANS SCP & 142 \\
DR1 SCP & 130 \\
PMMA & 100 \\
PS & 98 \\
PBMA & 15 \\
\hline
\end{tabular}

${ }^{a}$ Values are given for undoped host polymers and for the two SCP's that we investigated.

extrapolated values of the absorption coefficient at $775 \mathrm{~nm}$. For example, there is a difference of 2 orders of magnitude between the predicted absorption coefficient of 5 in PS C $\left(0.2 \mathrm{~cm}^{-1}\right)$ and the predicted absorption coefficient in PES E $\left(10.2 \mathrm{~cm}^{-1}\right)$.

One can relate the previous results to a specific macroscopic parameter characteristic of the different matrices. The glass transition temperature $T_{g}$ of this series of matrices varied from 15 to $300{ }^{\circ} \mathrm{C}$ as reported in Table 2, which values correspond to neat polymers (the relatively small chromophore loading should not significantly affect these values). If we exclude the result for the PBMA polymer $\mathrm{B}\left(T_{\sigma}=\right.$ $15{ }^{\circ} \mathrm{C}$ ), a surprising correlation was found when this slope, or the extrapolated absorption at $775 \mathrm{~nm}$, was plotted versus the $T_{g}$ of the solid matrices, as shown in Fig. 8. An approximately linear behavior is evident. The low- $T_{g}$ result (PBMA) does not follow this linear behavior because that absorption measurement was performed at room temperature (i.e., slightly above the $T_{g}$ of this polymer). Additional experiments are currently being carried out to gain better characterization and understanding of this apparent relationship between loss and $T_{g}$.

\section{Discussion}

We interpret these data in terms of a matrixdependent inhomogeneous spectral broadening of the absorption spectrum that is due to the different interactions that occur between the organic chromophores and the different matrix environments. The higher the $T_{g}$, the more rigid the matrix and the larger the range of different interactions or conformations imposed by the environment onto the chromophore. In a similar manner, it can be observed that the larger the acceptor or donor groups, the larger the impact on the inhomogeneous broadening. For example, in Table 1 one can see that chromophores with a diphenyl donor group (as in 15, 16, and 18) exhibit smaller slopes than chromophores with a dialkyl donor group $(13,14$, and 17 , respectively).

This behavior clearly identifies a new trade-off that must be taken into account for photonic devices. Currently there is a research thrust to increase the 

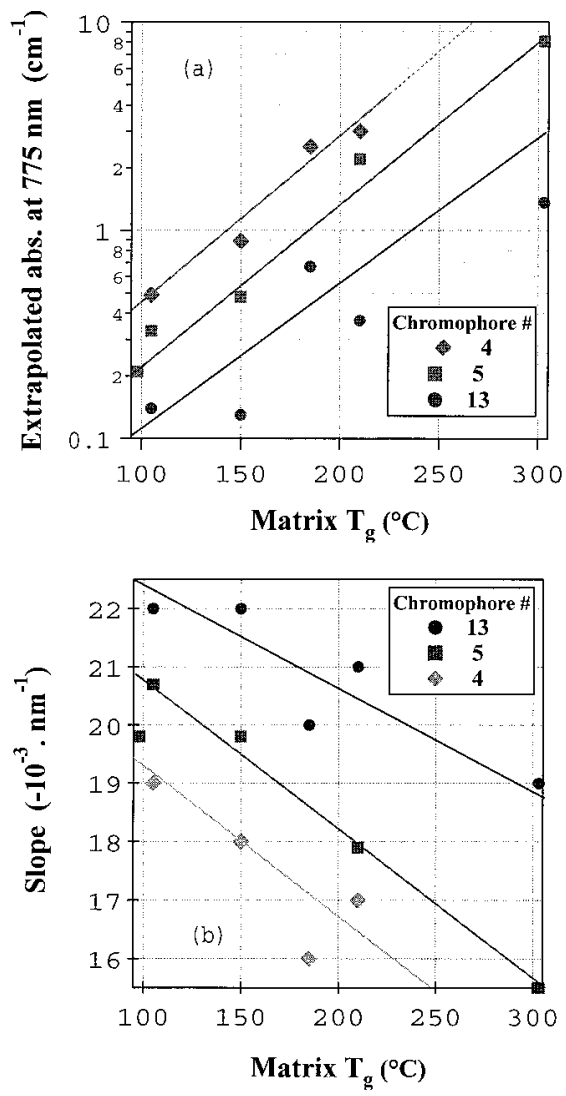

Fig. 8. Effect of the glass transition temperature $T_{g}$ on extrapolated values of the absorption at $775 \mathrm{~nm}$ and on the slope for the three different chromophores 4, 5, and 13. Chromophore aggregation prevented us from reporting the values for both 4 and 13 in polystyrene. (a) Extrapolated values of the absorption at $775 \mathrm{~nm}$ as a function of matrix $T_{g}$ (straight lines are guides for the eye). The losses increase by more than an order of magnitude as $T_{g}$ varies from approximately $100{ }^{\circ} \mathrm{C}$ to $300{ }^{\circ} \mathrm{C}$. The identities of the different polymers are listed in Table 2. (b) Values of the slope in the near-infrared tail of chromophore absorption as a function of matrix $T_{g}$. Note the following correlation: the larger the $T_{g}$, the smaller the slope (straight lines are guides for the eye). The identities of the different polymers are listed in Table 2.

$T_{g}$ of the polymer matrix to decrease the chromophore's polar orientational mobility and thus increase the long-term stability of the nonlinear coefficient. ${ }^{11,12}$ However, as shown here, this could lead to a simultaneous increase in the absorption losses. This effect poses a new challenge, namely, to develop new polymer matrices, organic or hybrid, that would induce as little as possible inhomogeneous spectral broadening of the main absorption band. The results given here show that a reduction of more than an order of magnitude in absorption losses can be achieved by fine tuning the chromophore environment. Therefore, within a family of chromophores, it should be possible to use chromophores with the largest nonlinearities whose $\lambda_{\max }$ is typically redshifted by finding complementary polymeric environments to reduce the absorptive losses. Thus the nonlinearity loss trade-off could conceivably be increased by as much as an order of magnitude.

\section{Conclusion}

The long-wavelength, near-infrared tail of the absorption spectrum of electro-optic chromophores appears to be extremely sensitive to the specific environment in the polymer matrix. In contrast to the slight change in intensity and width of the main absorption bands that are observed in the visible when changing from solution to solid, a dramatic difference in the absorption intensity has been found in the near-infrared spectral region. A correlation to the $T_{g}$ of the host polymer has been observed (although other influences could be operating as well). These results are important in view of optimizing doped polymers for applications in waveguided optics by use of red or near-infrared wavelengths. Furthermore, finding effective means of reducing such unnecessary absorption also affects the material lifetime associated with photodegradation. ${ }^{13,14}$ This research suggests that further characterization and analysis needs to be pursued to optimize the nonlinearity loss trade-off. Also, the impact of grafting the chromophore into side-chain polymer systems should be quantified. We expect such effort to lead to better doped-polymer materials.

This research was supported in the United States by a Ballistic Missile Defense Organization, Small Business Technology Transfer grant F29601-98-C0205. We also gratefully acknowledge Akzo-Nobel for providing us with the DANS SCP material. M. Canva and T. Pliska acknowledge partial financial support by, respectively, a NATO Science Fellowship and the Swiss National Science Foundation. This research was also supported by the National Science Foundation and the Centre National de la Recherche Scientifique U.S. and French bilateral collaboration funds.

\section{References and Notes}

1. As in the Proceedings of the Plastic Optical Fibers International Conference, San Jose 28-30 June 99, www.pofig.com POF Interest Group.

2. Y. Koike, Y. T. Ishigure, T. M. Satoh, and E. Nihei, "High-speed photonics polymer and its application," Pure Appl. Opt. 7, 201-210 (1998).

3. D. Chen, H. Fetterman, A. Chen, W. Steier, L. Dalton, W. Wang, and Y. Shi, "Demonstration of $110 \mathrm{GHz}$ electro-optic polymer modulators," Appl. Phys. Lett. 70, 3335-3337 (1997).

4. As reviewed in Nonlinear Optics of Organic Molecules and Polymers, H. S. Nalwa and S. Miyata, eds. (CRC Press, Boca Raton, Fla., 1996), and references therein.

5. G. Stegeman, D. Hagan and L. Torner, " $\chi{ }^{(2)}$ cascading phenomena and their applications to all-optical signal processing, mode locking, pulse compression and solitons," Opt. Quantum Electron. 28, 1691-1740 (1996).

6. A. Otomo, M. Jäger, G. Stegeman, M. Flipse, and M. Diemeer, "Key trade-offs for second harmonic generation in poled polymers," Appl. Phys. Lett. 69, 1991-1993 (1996).

7. J. Oudar, "Optical nonlinearities of conjugated molecules. Stilbene derivatives and highly polar aromatic compounds," J. Chem. Phys. 67, 446-456 (1977).

8. K. Singer, M. Kuzyk, and J. Söhn, "Second order nonlinear 
optical processes in orientationally ordered materials: relationship between molecular and macroscopic properties," J. Opt. Soc. Am. B 4, 968-976 (1987).

9. The guest chromophores were all prepared and characterized by standard methods. Many of these chromophores were originally prepared for use in studies of thermal stability: R. B. Prime, G. Y. Chiou, and R. J. Twieg, "Evaluation of the thermal stability of some nonlinear optical chromophores," J. Therm. Anal. 46, 1133-1150 (1996); R. J. Twieg and C. W. Dirk, "Design, properties and applications of nonlinear optical chromophores," in Organic Thin Films for Waveguiding Nonlinear Optics, F. Kajzar and J. D. Swalen, eds. (Gordon \& Breach, Newark, N.J., 1996).

10. N. Berard, M. Paventi, K. P. Chan, and A. S. Hay, "Polymers from 4-(4-hydroxyphenyl)phthalazin-1-one," Makromol. Chem. Macromol. Symp. 77, 379-388 (1994).
11. D. Jungbauer, I. Teraoka, D. Yoon, B. Reck, J. Swalen, R. Twieg, and C. Willson, "Second-order nonlinear optical properties and relaxation characteristics of poled linear epoxy polymers with tolane chromophores," J. Appl. Phys. 69, 8011-8017 (1991).

12. R. Jeng, Y. Chen, A. Jain, J. Kumar, and S. Tripathy, "Stable second-order nonlinear optical polyimide/inorganic composite," Chem. Mater. 4, 1141-1144 (1992).

13. Q. Zhang, M. Canva, and G. Stegeman, "Wavelength dependence of 4-dimethylamino-4'-nitrostilbene polymer thin film photodegradation,” Appl. Phys. Lett. 73, 912-914 (1998).

14. A. Galvan-Gonzales, M. Canva, G. Stegeman, S. Marder, S. Thayumanavan, R. Twieg, T. Kowalczyk, X. Zhang, and H. Lackritz, "Systematics of the wavelength dependence of electro-optic chromophore photostability," Opt. Lett. (to be published). 